\title{
On Two Classes of Extended 3-Lie Algebras
}

\author{
Yu Cheng1, Yansha Gao \\ ${ }^{1}$ School of Data Science and Software Engineering, Baoding University, Baoding, China \\ ${ }^{2}$ College of Mathematics and Information Science, Hebei University, Baoding, China \\ Email: chengyu72@sina.com
}

How to cite this paper: Cheng, Y. and Gao, Y.S. (2021) On Two Classes of Extended 3-Lie Algebras. Journal of Applied Mathematics and Physics, 9, 834-845. https://doi.org/10.4236/jamp.2021.94056

Received: February 24, 2021

Accepted: April 27, 2021

Published: April 30, 2021

Copyright $\odot 2021$ by author(s) and Scientific Research Publishing Inc. This work is licensed under the Creative Commons Attribution International License (CC BY 4.0).

http://creativecommons.org/licenses/by/4.0/

\begin{abstract}
In this paper, based on the existing research results, we obtain the unary extension 3-Lie algebras by one-dimensional extension of the known Lie algebra $L$. For two known 3-Lie algebras $H, M$, the $(\mu, \rho, \beta)$-extension of $H$ through $M$ is given, and the necessary and sufficient conditions for the $(\mu, \rho, \beta)$ -extension algebra of $H$ through $M$ being 3-Lie algebra are obtained, and the structural characteristics and properties of these two kinds of extended 3-Lie algebras are given.
\end{abstract}

\section{Keywords}

The Unary Extension 3-Lie Algebras, Lie Algebra, $(\mu, \rho, \beta)$-Extension

\section{Introduction}

In recent years, the study of 3-Lie algebra has been paid much attention because of its wide application in mathematics and physics. 3-Lie algebra is a special form of $n$-Lie algebra, which is an algebraic system with ternary linearly oblique symmetric multiplication table satisfying the generalized Jacobi equation [1]. 3-Lie algebra has extremely profound and rich algebraic and analytical structure. In this paper, the extension problem of 3-Lie algebra is studied on the basis of the existing research. Firstly, we define the unary extended 3-Lie algebra for a known Lie algebra $L$ by one-dimensional extension, and study its properties. Secondly, for two known 3-Lie algebras $H, M$, the $(\mu, \rho, \beta)$-extension of $H$ through $M$ is defined, and the $(\mu, \rho, \beta)$-extension of $H$ through $M$ is given as a necessary and sufficient condition for the 3-Lie algebra. Finally, the structure and properties of this extended 3-Lie algebra are discussed. Thus, it lays a foundation for the further study of the properties of the derivatives of two kinds of 3-Lie algebras. 


\section{Fundamental Notions}

Firstly, the basic knowledge [1]-[9] to be used in this paper is given.

Definition 2.1 Let $A$ be a vector space over a domain $F$ and have a 3-element linear operation $[,]:, A \wedge A \wedge A \rightarrow A$, satisfied for arbitrary, $x_{1}, x_{2}, x_{3}, y_{2}, y_{3} \in A$

$$
\left[\left[x_{1}, x_{2}, x_{3}\right], y_{2}, y_{3}\right]=\sum_{i=1}^{3}\left[x_{1},\left[x_{i}, y_{2}, y_{3}\right], x_{3}\right] \text {, }
$$

$(A,[,]$,$) is called 3-Lie algebra. Without confusion, A$ is called 3-Lie algebra for short.

Definition 2.2 Let $A$ be a 3-Lie algebra, and $D$ be a linear transformation of $A$, if this equation is satisfied

$$
[D(x), y, z]+[x, D(y), z]+[x, y, D(z)]=D([x, y, z]), x, y, z \in A
$$

Then $D$ is the derivative of $A$, and the set of derivatives is denoted by $\operatorname{Der}(A)$. It is easy to prove that $\operatorname{Der}(A)$ is a subalgebra of the general linear Lie algebra $g l(A)$.

The map

$$
\operatorname{ad}\left(x_{1}, x_{2}\right): A \rightarrow A, \quad a d\left(x_{1}, x_{2}\right)(x)=\left[x_{1}, x_{2}, x\right]
$$

for $x \in A$ is called the left multiplication defined by elements $x_{1}, x_{2} \in A$. Obviously the left multiplication is the derivative. The linear combination of the left multiplication is called the inner derivative, denoted by $a d(A)$.

Let $B$ be a subspace of $A$, and if $[B, B, B] \subseteq B([B, A, A] \subseteq B)$, then $B$ be a subalgebra (ideal) of $A$. And if $[B, B, B]=0([B, B, A]=0)$, then $B$ is called a $A b e l$ subalgebra

( $A b e l$ ideal). In particular, the subalgebra spanned by $\left[x_{1}, x_{2}, x_{3}\right]\left(\forall x_{1}, x_{2}, x_{3} \in A\right)$ is called the derivative algebra of $A$, denoted by $A^{1}$. If $A^{1}=0$, then $A$ is called $A b e l$ algebra. If an ideal $I$ of $A$ is a $A b e l$ subalgebra but not an $A b e l$ ideal, that is $[I, I, I]=0$, but $[I, I, A] \neq 0$, then $I$ is called an hypo-abelian ideal.

The ideal $I$ of a 3 -Lie algebra $A$ is called $s$-solvable, $2 \leq s \leq 3$, if $I^{(k, s)}=0$ for some $k \geq 0$, where $I^{(0, s)}=I, I^{(k+1, s)}$ is defined as

$I^{(k+1, s)}=[\underbrace{I^{(k, s)}, \cdots, I^{(k, s)}}_{s}, \underbrace{A, \cdots, A}_{3-s}]$. Where 2-solvable is also called solvable, and $I^{(k, s)}$ is abbreviated as $I^{(k)}$.

An ideal $I$ of a 3-Lie algebra $A$ is called nilpotent if $I^{s}=0$ for some $s \geq 0$, where $I^{0}=I$ and $I^{s}=\left[I^{s-1}, I, A\right]$.

The center of $A$ is denoted by $Z(A)=\{x \in A \mid[x, A, A]=0\}$. Obviously $Z(A)$ is the $A b e l$ ideal of $A$.

Let $A$ is a 3-Lie algebra over the field $F, V$ is a vector space, $\rho: A \wedge A \rightarrow \operatorname{End}(V)$ is a linear mapping, if $\rho$ satisfies for any $x_{1}, x_{2}, x_{3}, x_{4} \in A$

$$
\begin{gathered}
{\left[\rho\left(x_{1}, x_{2}\right), \rho\left(x_{3}, x_{4}\right)\right]=\rho\left(\left[x_{1}, x_{2}, x_{3}\right] x_{4}\right)-\rho\left(\left[x_{1}, x_{2}, x_{4}\right] x_{3}\right),} \\
\rho\left(\left[x_{1}, x_{2}, x_{3}\right], x_{4}\right)=\rho\left(x_{1}, x_{2}\right) \rho\left(x_{3}, x_{4}\right)+\rho\left(x_{2}, x_{3}\right) \rho\left(x_{1}, x_{4}\right) \\
+\rho\left(x_{3}, x_{1}\right) \rho\left(x_{2}, x_{4}\right)
\end{gathered}
$$


Then $(V, \rho)$ is called the representation of $A$ (or $(V, \rho)$ is $A$-module).

Lemma 2.1 Let $A$ is a 3-Lie algebra over the field $F, V$ is a vector space, $\rho: A \wedge A \rightarrow \operatorname{End}(V)$ is a linear mapping. If $(V, \rho)$ is an $A$-module, then for any $x, y, z, u \in A$, the following equation is true:

$$
\begin{aligned}
& \rho([x, y, z], u)-\rho([x, y, u], z)+\rho([x, z, u], y)-\rho([y, z, u], x)=0, \\
& \rho(x, u) \rho(y, z)+\rho(y, z) \rho(x, u)+\rho(x, y) \rho(z, u)+\rho(z, u) \rho(x, y) \\
& -\rho(x, z) \rho(y, u)-\rho(y, u) \rho(x, z)=0 .
\end{aligned}
$$

\section{The Unary Extension 3-Lie Algebra of Lie Algebras}

Definition 3.1 Let $(L,[]$,$) be a Lie algebra over a field F$, let $A=L \oplus F x_{0}$ $x_{0} \in F$, and $x_{0} \notin L$. Linear operation $[,]:, A \wedge A \wedge A \rightarrow A$ for all $x, y, z \in L$ that satisfy the following multiplication table:

$$
\left[x, y, x_{0}\right]=[x, y],[x, y, z]=0 .
$$

Then $A$ is called the unary extension of Lie algebra $L$. If $(A,[,]$,$) is a 3-Lie$ algebra, then $(A,[,]$,$) is a unary extension 3-Lie algebra of the Lie algebra L$.

Lemma 3.1 let $L$ be a Lie algebra over a field $F$. If let $A=L \oplus F x_{0} \quad x_{0} \in F$, $x_{0} \notin L$ and the multiplication of is defined by (7), then $A$ is a 3-Lie algebra, and for positive integers $m$, the following equation holds

$$
A^{(m)}=L^{(m)}, A^{(m, 2)}=L^{(m, 2)}=L^{(m)}, A^{(2,3)}=0 .
$$

Proof: By multiplication (7), direct calculation $A$ is 3-Lie algebra. Due to the

$$
\begin{gathered}
A^{1}=[A, A, A]=[L, L, L]+\left[L, L, F x_{0}\right]=L^{1}, \\
A^{2}=\left[A^{1}, A, A\right]=\left[L^{1}, L, F x_{0}\right]=L^{2},
\end{gathered}
$$

Assume $A^{m-1}=L^{m-1}$, then

$$
A^{m}=\left[A^{m-1}, A, A\right]=\left[L^{m-1}, L+F x_{0}, L+F x_{0}\right]=\left[L^{m-1}, L\right]=L^{m} .
$$

similarly, $A^{(m, 2)}=L^{(m, 2)}=L^{(m)}$ and $A^{(2,3)}=0$. The conclusion is proved.

Theorem 3.1 Let $L$ be a Lie algebra on the field $F$ and $A=L \oplus F x_{0}$ be a unary extension 3-Lie algebra, where $x_{0} \in F$ and $x_{0} \notin L$, then

1) $A$ is 2 -solvable if and only if $L$ is a solvable Lie algebra.

2) $A$ is nilpotent if and only if $L$ is a nilpotent Lie algebra.

3) $A$ is 3-solvable.

4) $Z(A)=Z(L)$.

Proof: According to lemma 3.1, (1), (2) and (3) can be obtained directly. It is proved below that (4) is true. If $L^{1}=0$, then $A^{1}=L^{1}=0$ and $Z(A)=Z(L)$. If $L^{1} \neq 0$, then exists $y, z \in L$ such that $[y, z] \neq 0$. For any $x \in L, \lambda \in F$, $x+\lambda x_{0} \in Z(A)$, because of $\left[x+\lambda x_{0}, y, z\right]=\lambda[y, z]=0$, therefore $\lambda=0$.And because $\left[x+\lambda x_{0}, A, x_{0}\right]=[x, L]=0$, so $x \in Z(L)$. Therefore $Z(A) \subseteq Z(L)$. Obviously, the conclusion of $Z(L) \subseteq Z(A)$ is true.

Theorem 3.2 Let $L$ be a Lie algebra on the field $F$ and $I$ be a subspace of $L$ :

1) $I$ is an ideal of $A$ if and only if $I$ is an ideal of $L$. 
2) Let $J=I \oplus F x_{0}$, then $J$ is ideal of $A$ if and only if $L^{1} \subseteq I$.

3) If $L^{1} \subseteq I$, then for positive integers $m, J^{(m, 2)} \subseteq I^{(m-1)}$. If $I$ is a solvable ideal of $L$, then $J$ is a 2 -solvable ideal of $A$.

4) If $L$ is a simple Lie algebra, then $L$ is hypo-abelian ideal of $A$.

Proof: From $[I, A, A]=\left[I, L, x_{0}\right]=[I, L]$, we can get (1). From Equation (7),

$$
[J, A, A]=\left[I, L, x_{0}\right]+\left[x_{0}, L, L\right]=[I, L]+[L, L],
$$

So $[J, A, A] \subseteq J$ if and only if $[L, L] \subseteq I$. That means (2) is true.

If $I$ is the ideal of $L$ and $L^{1} \subseteq I$, then

$$
\begin{gathered}
J^{(1,2)}=[J, J, A]=[I, I]+[I, L]=I^{(1)}+[I, L] \subseteq I^{(1)}+I \subseteq I=I^{(0)}, \\
J^{(2,2)}=\left[J^{(1,2)}, J^{(1,2)}, A\right]=\left[I, I, L+F x_{0}\right] \subseteq I^{(1)},
\end{gathered}
$$

Assuming $J^{(m-1,2)} \subseteq I^{(m-2)}$ is true, then

$$
J^{(m, 2)}=\left[J^{(m-1,2)}, J^{(m-1,2)}, A\right] \subseteq\left[I^{(m-2)}, I^{(m-2)}, L+F x_{0}\right] \subseteq I^{(m-1)} .
$$

Therefore (3) holds. If $L$ is a simple Lie algebra, then $L$ is ideal of $A$, and $[L, L, L]=0,[L, L, A]=\left[L, L, x_{0}\right]=L^{1} \neq 0$. Therefore, $L$ is hypo-abelian ideal of $A$. That's the end of the argument.

\section{4. $(\mu, \rho, \beta)$-Extension of 3 -Lie Algebras}

Definition 4.1 Let $\left(H,[,,]_{H}\right)$ and $\left(M,[,,]_{M}\right)$ be two 3-Lie algebras over the field $F, A=M \oplus H$, and

$$
\rho: M \wedge M \rightarrow \operatorname{Der}(H), \quad \beta: M \wedge H \rightarrow \operatorname{Der}(H), \quad \mu: M \wedge M \wedge M \rightarrow H
$$

is linear mappings. Define a linear operation $[,,]_{\mu \rho \beta}: A \wedge A \wedge A \rightarrow A$, for any $x, y, z \in M, h, h_{1}, h_{2} \in H$ that satisfies the multiplication table:

$$
\begin{gathered}
{[x, y, z]_{\mu \rho \beta}=[x, y, z]_{M}+\mu(x, y, z),[x, y, h]_{\mu \rho \beta}=\rho(x, y) h} \\
{\left[h_{1}, h_{2}, h_{3}\right]_{\mu \rho \beta}=\left[h_{1}, h_{2}, h_{3}\right]_{H},\left[x, h_{1}, h_{2}\right]_{\mu \rho \beta}=\beta\left(x, h_{1}\right) h_{2} .}
\end{gathered}
$$

Then $\left(A,[,,]_{\mu \rho \beta}\right)$ is called the $(\mu, \rho, \beta)$-extension of $H$ through $M$. If $\left(A,[,,]_{\mu \rho \beta}\right)$ is a 3 -Lie algebra, then $\left(A,[,,]_{\mu \rho \beta}\right)$ is $(\mu, \rho, \beta)$-extension algebra of 3-Lie algebra. If $\beta=0$, then $A$ is called $(\mu, \rho)$-extension of $H$ through $M$, and $[,,]_{\mu \rho \beta}$ denoted as $[,,]_{\mu \rho}$. For convenience, we will abbreviate $[,,]_{M}$ and $[,,]_{H}$ as $[,$,$] and [,,]_{\mu \rho \beta}$ as $[,,]_{A}$.

Lemma 4.1 Let $\left(H,[,,]_{H}\right)$ and $\left(M,[,,]_{M}\right)$ be two 3-Lie algebras over the field $F$, and $A$ be the $(\mu, \rho, \beta)$-extension of $H$ through $M$, and for all $x_{1}, x_{2}, x_{3}, x_{4} \in M$ satisfy

$$
\begin{aligned}
\rho\left(x_{4},\left[x_{1}, x_{2}, x_{3}\right]\right)= & \rho\left(x_{3}, x_{1}\right) \rho\left(x_{4}, x_{2}\right)-\rho\left(x_{2}, x_{1}\right) \rho\left(x_{4}, x_{3}\right) \\
& +\rho\left(x_{2}, x_{3}\right) \rho\left(x_{4}, x_{1}\right)-\beta\left(x_{4}, \mu\left(x_{1}, x_{2}, x_{3}\right)\right) .
\end{aligned}
$$

Then Equation (6) is true if and only if the following equation 


$$
\begin{aligned}
& \rho\left(x_{4},\left[x_{1}, x_{2}, x_{3}\right]\right) \\
& =\rho\left(x_{3},\left[x_{1}, x_{2}, x_{4}\right]\right)-\beta\left(x_{4}, \mu\left(x_{1}, x_{2}, x_{3}\right)\right)+\beta\left(x_{3}, \mu\left(x_{1}, x_{2}, x_{4}\right)\right) \\
& -\rho\left(x_{1}, x_{2}\right) \rho\left(x_{3}, x_{4}\right)+\rho\left(x_{3}, x_{4}\right) \rho\left(x_{1}, x_{2}\right) .
\end{aligned}
$$

Proof: From Equation (9), we can get

$$
\begin{aligned}
& \quad \rho\left(x_{3},\left[x_{1}, x_{2}, x_{4}\right]\right)=\rho\left(x_{2}, x_{4}\right) \rho\left(x_{3}, x_{1}\right)-\rho\left(x_{1}, x_{4}\right) \rho\left(x_{3}, x_{2}\right) \\
& +\rho\left(x_{1}, x_{2}\right) \rho\left(x_{3}, x_{4}\right)-\beta\left(x_{3}, \mu\left(x_{1}, x_{2}, x_{4}\right)\right), \\
& \rho\left(x_{4},\left[x_{1}, x_{2}, x_{3}\right]\right)-\rho\left(x_{3},\left[x_{1}, x_{2}, x_{4}\right]\right) \\
& =\rho\left(x_{1}, x_{3}\right) \rho\left(x_{2}, x_{4}\right)-\rho\left(x_{1}, x_{2}\right) \rho\left(x_{3}, x_{4}\right)-\rho\left(x_{2}, x_{3}\right) \rho\left(x_{1}, x_{4}\right) \\
& -\beta\left(x_{4}, \mu\left(x_{1}, x_{2}, x_{3}\right)\right)+\rho\left(x_{2}, x_{4}\right) \rho\left(x_{1}, x_{3}\right)-\rho\left(x_{1}, x_{4}\right) \rho\left(x_{2}, x_{3}\right) \\
& -\rho\left(x_{1}, x_{2}\right) \rho\left(x_{3}, x_{4}\right)+\beta\left(x_{3}, \mu\left(x_{1}, x_{2}, x_{4}\right)\right) \\
& =\rho\left(x_{1}, x_{3}\right) \rho\left(x_{2}, x_{4}\right)+\rho\left(x_{2}, x_{4}\right) \rho\left(x_{1}, x_{3}\right)-\rho\left(x_{2}, x_{3}\right) \rho\left(x_{1}, x_{4}\right) \\
& -
\end{aligned}
$$

So Equation (10) holds. On the other hand, if

$$
\begin{aligned}
\rho\left(x_{4},\left[x_{1}, x_{2}, x_{3}\right]\right)= & \rho\left(x_{2}, x_{4}\right) \rho\left(x_{3}, x_{1}\right)-\rho\left(x_{1}, x_{4}\right) \rho\left(x_{3}, x_{2}\right) \\
& +\rho\left(x_{1}, x_{2}\right) \rho\left(x_{3}, x_{4}\right)-\beta\left(x_{3}, \mu\left(x_{1}, x_{2}, x_{4}\right)\right) \\
& -\beta\left(x_{4}, \mu\left(x_{1}, x_{2}, x_{3}\right)\right)+\beta\left(x_{3}, \mu\left(x_{1}, x_{2}, x_{4}\right)\right) \\
& -\rho\left(x_{1}, x_{2}\right) \rho\left(x_{3}, x_{4}\right)+\rho\left(x_{3}, x_{4}\right) \rho\left(x_{1}, x_{2}\right) .
\end{aligned}
$$

Through Equation (9), it can be concluded that Equation (6) holds.

Lemma 4.2. Let $A$ be the $(\mu, \rho, \beta)$-extension of $H$ through $M$, for all $x_{1}, x_{2} \in M, h_{1}, h_{2}, h \in H$ satisfies

$$
\begin{aligned}
& \beta\left(y, h_{2}\right) \beta\left(x, h_{1}\right) h-\beta(y, h) \beta\left(x, h_{1}\right) h_{2}-\beta\left(x, h_{1}\right) \beta\left(y, h_{2}\right) h \\
& =\left[\rho(x, y) h_{1}, h_{2}, h\right]
\end{aligned}
$$

There are

$$
\begin{aligned}
& \rho(x, y)\left[h_{1}, h_{2}, h\right]+\beta\left(y, h_{1}\right) \beta\left(x, h_{2}\right) h-\beta\left(x, h_{1}\right) \beta\left(y, h_{2}\right) h \\
& =\left[\rho(x, y) h_{1}, h_{2}, h\right]
\end{aligned}
$$

Proof: From Equation (11) and the $\rho(x, y)$ is derivative of $H$, we can get

$$
\begin{aligned}
& \quad\left[h_{1}, \rho(x, y) h_{2}, h\right] \\
& =\beta\left(y, h_{1}\right) \beta(x, h) h_{2}+\beta(y, h) \beta\left(x, h_{2}\right) h_{1}+\beta\left(x, h_{2}\right) \beta\left(y, h_{1}\right) h \\
& \quad\left[h_{1}, h_{2}, \rho(x, y) h\right] \\
& =\beta\left(y, h_{2}\right) \beta\left(x, h_{1}\right) h+\beta\left(y, h_{1}\right) \beta(x, h) h_{2}+\beta(x, h) \beta\left(y, h_{2}\right) h_{1} \\
& \rho(x, y)\left[h_{1}, h_{2}, h\right] \\
& =2\left(\beta\left(y, h_{1}\right) \beta(x, h) h_{2}+\beta\left(y, h_{2}\right) \beta\left(x, h_{1}\right) h+\beta(y, h) \beta\left(x, h_{2}\right) h_{1}\right) \\
& +\beta\left(x, h_{1}\right) \beta(y, h) h_{2}+\beta\left(x, h_{2}\right) \beta\left(y, h_{1}\right) h+\beta(x, h) \beta\left(y, h_{2}\right) h_{1},
\end{aligned}
$$




$$
\begin{aligned}
& \beta\left(y, h_{1}\right) \beta(y, h) h_{2}+\beta\left(y, h_{2}\right) \beta\left(x, h_{1}\right) h+\beta(y, h) \beta\left(x, h_{2}\right) h_{1} \\
& +\beta\left(x, h_{1}\right) \beta(y, h) h_{2}+\beta\left(x, h_{2}\right) \beta\left(y, h_{1}\right) h+\beta(y, h) \beta\left(y, h_{2}\right) h_{1}=0, \\
& \quad \rho(x, y)\left[h_{1}, h_{2}, h\right] \\
& \quad=\beta\left(y, h_{1}\right) \beta(x, h) h_{2}+\beta\left(y, h_{2}\right) \beta\left(x, h_{1}\right) h+\beta(y, h) \beta\left(x, h_{2}\right) h_{1}
\end{aligned}
$$

Namely

$$
\begin{aligned}
& \beta\left(y, h_{2}\right) \beta\left(x, h_{1}\right) h-\beta(y, h) \beta\left(x, h_{1}\right) h_{2} \\
& =\rho(x, y)\left[h_{1}, h_{2}, h\right]+\beta\left(y, h_{1}\right) \beta\left(x, h_{2}\right) h .
\end{aligned}
$$

Using Equation (11) again, Equation (12) can be obtained.

Lemma 4.3. Let $A$ be the $(\mu, \rho, \beta)$-extension of $H$ through $M$. If for all $x \in M$, $h_{1}, h_{2}, h_{3}, h_{4} \in H$ satisfies

$$
\begin{aligned}
& \operatorname{ad}\left(\beta\left(x, h_{1}\right) h_{3}, h_{2}\right)+a d\left(h_{3}, \beta\left(x, h_{1}\right) h_{2}\right)+a d\left(\beta\left(x, h_{3}\right) h_{2}, h_{1}\right) \\
& =\beta\left(x,\left[h_{1}, h_{2}, h_{3}\right]\right),
\end{aligned}
$$

Then

$$
\begin{aligned}
& {\left[h_{1}, h_{2}, \beta\left(x, h_{3}\right) h_{4}\right]-\beta\left(x,\left[h_{1}, h_{2}, h_{3}\right]\right) h_{4}-\left[h_{3}, h_{4}, \beta\left(x, h_{1}\right) h_{2}\right]} \\
& =\beta\left(x, h_{3}\right)\left[h_{1}, h_{2}, h_{4}\right] .
\end{aligned}
$$

Proof: According to Equation (13),

$$
\begin{aligned}
& \beta\left(x,\left[h_{1}, h_{2}, h_{3}\right]\right) h_{4} \\
& =\left[\beta\left(x, h_{1}\right) h_{3}, h_{2}, h_{4}\right]+\left[h_{3}, \beta\left(x, h_{1}\right) h_{2}, h_{4}\right]+\left[\beta\left(x, h_{3}\right) h_{2}, h_{1}, h_{4}\right] .
\end{aligned}
$$

Because of $\beta\left(x, h_{3}\right) \in \operatorname{Der}(H)$, therefore

$$
\begin{aligned}
& \beta\left(x,\left[h_{1}, h_{2}, h_{3}\right]\right) h_{4} \\
& =\left[\beta\left(x, h_{1}\right) h_{3}, h_{2}, h_{4}\right]+\left[h_{3}, \beta\left(x, h_{1}\right) h_{2}, h_{4}\right]+\left[\beta\left(x, h_{3}\right) h_{2}, h_{1}, h_{4}\right] \\
& =-\left[\beta\left(x, h_{3}\right) h_{1}, h_{2}, h_{4}\right]+\left[h_{3}, \beta\left(x, h_{1}\right) h_{2}, h_{4}\right]+\left[\beta\left(x, h_{3}\right) h_{2}, h_{1}, h_{4}\right] \\
& =-\beta\left(x, h_{3}\right)\left[h_{1}, h_{2}, h_{4}\right]+\left[h_{1}, h_{2}, \beta\left(x, h_{3}\right) h_{4}\right]+\left[h_{3}, \beta\left(x, h_{1}\right) h_{2}, h_{4}\right] .
\end{aligned}
$$

Hence, Equation (14) holds.

Theorem 4.1. Let $A$ be the $(\mu, \rho, \beta)$-extension of $H$ through $M$, then $A$ is a 3-Lie algebra if and only if for any $x_{1}, x_{2}, x_{3}, x_{4}, x_{5} \in M, h_{1}, h_{2} \in H$, Equations (6), (9), (11), (13) and the following are true,

$$
\begin{aligned}
& \quad\left[\mu\left(x_{1}, x_{2}, x_{3}\right), h_{1}, h_{2}\right] \\
& =\rho\left(x_{2}, x_{3}\right) \beta\left(x_{1}, h_{1}\right) h_{2}-\rho\left(x_{1}, x_{3}\right) \beta\left(x_{2}, h_{1}\right) h_{2} \\
& \quad+\rho\left(x_{1}, x_{2}\right) \beta\left(x_{3}, h_{1}\right) h_{2}-\beta\left(\left[x_{1}, x_{2}, x_{3}\right], h_{1}\right) h_{2}, \\
& \beta\left(x_{1}, h_{1}\right) \rho\left(x_{2}, x_{3}\right) h_{2}+\beta\left(x_{3}, h_{2}\right) \rho\left(x_{1}, x_{2}\right) h_{1} \\
& =\rho\left(x_{2}, x_{3}\right) \beta\left(x_{1}, h_{1}\right) h_{2}+\beta\left(x_{2}, h_{2}\right) \rho\left(x_{1}, x_{3}\right) h_{1}, \\
& \mu\left(x_{1}, x_{2},\left[x_{3}, x_{4}, x_{5}\right]\right)-\mu\left(\left[x_{1}, x_{2}, x_{3}\right], x_{4}, x_{5}\right) \\
& -\mu\left(x_{3},\left[x_{1}, x_{2}, x_{4}\right], x_{5}\right)-\mu\left(x_{3}, x_{4},\left[x_{1}, x_{2}, x_{5}\right]\right) \\
& =\rho\left(x_{3}, x_{4}\right) \mu\left(x_{1}, x_{2}, x_{5}\right)-\rho\left(x_{3}, x_{5}\right) \mu\left(x_{1}, x_{2}, x_{4}\right) \\
& -\rho\left(x_{1}, x_{2}\right) \mu\left(x_{3}, x_{4}, x_{5}\right)+\rho\left(x_{4}, x_{5}\right) \mu\left(x_{1}, x_{2}, x_{3}\right) .
\end{aligned}
$$


Proof: If $A$ is a 3-Lie algebra, the Equations (11), (15), (16) and (17) are obtained from the Equations (1). The following proves that Equations (6), (9) and (13) are true.

For $x_{i} \in M, i=1,2,3,4, h \in H$, according to (8),

$$
\begin{gathered}
{\left[h, x_{1},\left[x_{2}, x_{3}, x_{4}\right]_{A}\right]_{A}} \\
=\left[h, x_{1},\left[x_{2}, x_{3}, x_{4}\right]\right]_{A}+\left[h, x_{1}, \mu\left(x_{2}, x_{3}, x_{4}\right)\right]_{A} \\
=\rho\left(x_{1},\left[x_{2}, x_{3}, x_{4}\right]\right) h+\beta\left(x_{1}, \mu\left(x_{2}, x_{3}, x_{4}\right)\right) h \\
{\left[\left[h, x_{1}, x_{2}\right]_{A}, x_{3}, x_{4}\right]_{A}+\left[x_{2},\left[h, x_{1}, x_{3}\right]_{A}, x_{4}\right]_{A}+\left[x_{2}, x_{3},\left[h, x_{1}, x_{4}\right]_{A}\right]_{A}} \\
=\rho\left(x_{3}, x_{4}\right) \rho\left(x_{1}, x_{2}\right) h-\rho\left(x_{2}, x_{4}\right) \rho\left(x_{1}, x_{3}\right) h+\rho\left(x_{2}, x_{3}\right) \rho\left(x_{1}, x_{4}\right) h
\end{gathered}
$$

As a result,

$$
\begin{aligned}
& \rho\left(x_{1},\left[x_{2}, x_{3}, x_{4}\right]\right)+\beta\left(x_{1}, \mu\left(x_{2}, x_{3}, x_{4}\right)\right) \\
& =\rho\left(x_{3}, x_{4}\right) \rho\left(x_{1}, x_{2}\right)-\rho\left(x_{2}, x_{4}\right) \rho\left(x_{1}, x_{3}\right)+\rho\left(x_{2}, x_{3}\right) \rho\left(x_{1}, x_{4}\right) .
\end{aligned}
$$

In the above formula, $x_{1}, x_{2}, x_{3}, x_{4}$ is replaced by $x_{4}, x_{1}, x_{2}, x_{3}$, and Equation (9) can be obtained.

$$
\begin{aligned}
& \text { Because, }\left[x_{1}, x_{2},\left[x_{3}, x_{4}, h\right]_{A}\right]_{A}=\rho\left(x_{1}, x_{2}\right) \rho\left(x_{3}, x_{4}\right) h \\
& \quad\left[\left[x_{1}, x_{2}, x_{3}\right]_{A}, x_{4}, h\right]_{A}+\left[x_{3},\left[x_{1}, x_{3}, x_{4}\right]_{A}, h\right]_{A}+\left[x_{3}, x_{4},\left[x_{1}, x_{2}, h\right]_{A}\right]_{A} \\
& =\rho\left(\left[x_{1}, x_{2}, x_{3}\right], x_{4}\right) h-\beta\left(x_{4}, \mu\left(x_{1}, x_{2}, x_{3}\right)\right) h+\rho\left(x_{3},\left[x_{1}, x_{2}, x_{4}\right]\right) h \\
& \quad+\beta\left(x_{3}, \mu\left(x_{1}, x_{2}, x_{4}\right)\right) h+\rho\left(x_{3}, x_{4}\right) \rho\left(x_{1}, x_{2}\right) h .
\end{aligned}
$$

So Equation (10) holds. Equation (6) is obtained from lemma 4.1.

For arbitrary $h_{i} \in H, i=1,2,3,4, x \in M$, it can be known from (8) that,

$$
\begin{gathered}
{\left[h_{1}, h_{2},\left[h_{3}, h_{4}, x\right]_{A}\right]_{A}=\left[h_{1}, h_{2}, \beta\left(x, h_{3}\right) h_{4}\right],} \\
{\left[\left[h_{1}, h_{2}, h_{3}\right]_{A}, h_{4}, x\right]_{A}+\left[h_{3},\left[h_{1}, h_{2}, h_{4}\right]_{A}, x\right]_{A}+\left[h_{3}, h_{4}\left[h_{1}, h_{2}, x\right]_{A}\right]_{A}} \\
=\beta\left(x,\left[h_{1}, h_{2}, h_{3}\right]\right) h_{4}+\beta\left(x, h_{3}\right)\left[h_{1}, h_{2}, h_{4}\right]+\left[h_{3}, h_{4}, \beta\left(x, h_{1}\right) h_{2}\right],
\end{gathered}
$$

As a result,

$$
\begin{aligned}
& {\left[h_{1}, h_{2}, \beta\left(x, h_{3}\right) h_{4}\right]} \\
& =\beta\left(x,\left[h_{1}, h_{2}, h_{3}\right]\right) h_{4}+\beta\left(x, h_{3}\right)\left[h_{1}, h_{2}, h_{4}\right]+\left[h_{3}, h_{4}, \beta\left(x, h_{1}\right) h_{2}\right] .
\end{aligned}
$$

Because of $\beta\left(x, h_{3}\right) \in \operatorname{Der}(H)$, therefore

$$
\begin{aligned}
& \beta\left(x,\left[h_{1}, h_{2}, h_{3}\right]\right) h_{4} \\
& =\left[h_{1}, h_{2}, \beta\left(x, h_{3}\right) h_{4}\right]-\beta\left(x, h_{3}\right)\left[h_{1}, h_{2}, h_{4}\right]-\left[h_{3}, h_{4}, \beta\left(x, h_{1}\right) h_{2}\right] \\
& =-\left[\beta\left(x, h_{3}\right) h_{1}, h_{2}, h_{4}\right]-\left[h_{1}, \beta\left(x, h_{3}\right) h_{2}, h_{4}\right]+\left[h_{3}, \beta\left(x, h_{1}\right) h_{2}, h_{4}\right] \\
& =\operatorname{ad}\left(\beta\left(x, h_{1}\right) h_{3}, h_{2}\right) h_{4}+a d\left(h_{3}, \beta\left(x, h_{1}\right) h_{2}\right) h_{4}+\operatorname{ad}\left(\beta\left(x, h_{3}\right) h_{2}, h_{1}\right) h_{4} .
\end{aligned}
$$

Equation (13) holds.

Conversely, to prove that $A$ is a 3-Lie algebra, it is only necessary to prove that (8) satisfies Equation (1).

Case 1. For all $x_{i} \in M, i=1,2,3,4,5$, known by (8) 


$$
\begin{aligned}
& \quad\left[x_{1}, x_{2},\left[x_{3}, x_{4}, x_{5}\right]_{A}\right]_{A} \\
& =\left[x_{1}, x_{2},\left[x_{3}, x_{4}, x_{5}\right]\right]+\mu\left(x_{1}, x_{2},\left[x_{3}, x_{4}, x_{5}\right]\right)+\rho\left(x_{1}, x_{2}\right) \mu\left(x_{3}, x_{4}, x_{5}\right), \\
& {\left[\left[x_{1}, x_{2}, x_{3}\right]_{A}, x_{4}, x_{5}\right]_{A}+\left[x_{3},\left[x_{1}, x_{2}, x_{4}\right]_{A}, x_{5}\right]_{A}+\left[x_{3}, x_{4},\left[x_{1}, x_{2}, x_{5}\right]_{A}\right]_{A}} \\
& =\left[\left[x_{1}, x_{2}, x_{3}\right], x_{4}, x_{5}\right]+\mu\left(\left[x_{1}, x_{2}, x_{3}\right], x_{4}, x_{5}\right)+\rho\left(x_{4}, x_{5}\right) \mu\left(x_{1}, x_{2}, x_{3}\right) \\
& \quad+\left[x_{3},\left[x_{1}, x_{2}, x_{4}\right], x_{5}\right]+\mu\left(x_{3},\left[x_{1}, x_{2}, x_{4}\right], x_{5}\right)+\rho\left(x_{5}, x_{3}\right) \mu\left(x_{1}, x_{2}, x_{4}\right) \\
& \quad+\left[x_{3}, x_{4},\left[x_{1}, x_{2}, x_{5}\right]\right]+\mu\left(x_{3}, x_{4},\left[x_{1}, x_{2}, x_{5}\right]\right)+\rho\left(x_{3}, x_{4}\right) \mu\left(x_{1}, x_{2}, x_{5}\right) .
\end{aligned}
$$

From Equation (17), we can get

$$
\begin{aligned}
& {\left[x_{1}, x_{2},\left[x_{3}, x_{4}, x_{5}\right]_{A}\right]_{A}} \\
& =\left[\left[x_{1}, x_{2}, x_{3}\right]_{A}, x_{4}, x_{5}\right]_{A}+\left[x_{3},\left[x_{1}, x_{2}, x_{4}\right]_{A}, x_{5}\right]_{A}+\left[x_{3}, x_{4},\left[x_{1}, x_{2}, x_{5}\right]_{A}\right]_{A} .
\end{aligned}
$$

Case 2. For all $x_{i} \in M, i=1,2,3,4, h \in H$, know from (8)

$$
\begin{gathered}
{\left[h, x_{1},\left[x_{2}, x_{3}, x_{4}\right]_{A}\right]_{A}=\left[h, x_{1},\left[x_{2}, x_{3}, x_{4}\right]\right]_{A}+\left[h, x_{1}, \mu\left(x_{2}, x_{3}, x_{4}\right)\right]_{A}} \\
=\rho\left(x_{1},\left[x_{2}, x_{3}, x_{4}\right]\right) h+\beta\left(x_{1}, \mu\left(x_{2}, x_{3}, x_{4}\right)\right) h \\
{\left[\left[h, x_{1}, x_{2}\right]_{A}, x_{3}, x_{4}\right]_{A}+\left[x_{2},\left[h, x_{1}, x_{3}\right]_{A}, x_{4}\right]_{A}+\left[x_{2}, x_{3}\left[h, x_{1}, x_{4}\right]_{A}\right]_{A}} \\
=\rho\left(x_{3}, x_{4}\right) \rho\left(x_{1}, x_{2}\right) h-\rho\left(x_{2}, x_{4}\right) \rho\left(x_{1}, x_{3}\right) h+\rho\left(x_{2}, x_{3}\right) \rho\left(x_{1}, x_{4}\right) h .
\end{gathered}
$$

In Equation (9), by $x_{1}, x_{2}, x_{3}, x_{4}$ substitution for $x_{4}, x_{1}, x_{2}, x_{3}$, we can get

$$
\begin{aligned}
\rho\left(x_{1},\left[x_{2}, x_{3}, x_{4}\right]\right)= & \rho\left(x_{3}, x_{4}\right) \rho\left(x_{1}, x_{2}\right)-\rho\left(x_{2}, x_{4}\right) \rho\left(x_{1}, x_{3}\right) \\
& +\rho\left(x_{2}, x_{3}\right) \rho\left(x_{1}, x_{4}\right)-\beta\left(x_{1}, \mu\left(x_{2}, x_{3}, x_{4}\right)\right) .
\end{aligned}
$$

As a result,

$$
\begin{aligned}
& {\left[h, x_{1},\left[x_{2}, x_{3}, x_{4}\right]_{A}\right]_{A}} \\
& =\left[\left[h, x_{1}, x_{2}\right]_{A}, x_{3}, x_{4}\right]_{A}+\left[x_{2},\left[h, x_{1}, x_{3}\right]_{A}, x_{4}\right]_{A}+\left[x_{2}, x_{3},\left[h, x_{1}, x_{4}\right]_{A}\right]_{A} .
\end{aligned}
$$

Due to the $\left[x_{1}, x_{2},\left[x_{3}, x_{4}, h\right]_{A}\right]_{A}=\rho\left(x_{1}, x_{2}\right) \rho\left(x_{3}, x_{4}\right) h$,

$$
\begin{aligned}
& {\left[\left[x_{1}, x_{2}, x_{3}\right]_{A}, x_{4}, h\right]_{A}+\left[x_{3},\left[x_{1}, x_{2}, x_{4}\right]_{A}, h\right]_{A}+\left[x_{3}, x_{4},\left[x_{1}, x_{2}, h\right]_{A}\right]_{A}} \\
& =\rho\left(\left[x_{1}, x_{2}, x_{3}\right]_{A}, x_{4}\right) h-\beta\left(x_{4}, \mu\left(x_{1}, x_{2}, x_{3}\right)\right) h+\rho\left(x_{3},\left[x_{1}, x_{2}, x_{4}\right]\right) h \\
& +\beta\left(x_{3}, \mu\left(x_{1}, x_{2}, x_{4}\right)\right) h+\rho\left(x_{3}, x_{4}\right) \rho\left(x_{1}, x_{2}\right) h .
\end{aligned}
$$

Through lemma 4.1 and Equation (9), we can get

$$
\begin{aligned}
& \rho\left(x_{4},\left[x_{1}, x_{2}, x_{3}\right]\right)-\rho\left(x_{3},\left[x_{1}, x_{2}, x_{4}\right]\right) \\
& =\rho\left(x_{2}, x_{3}\right) \rho\left(x_{4}, x_{1}\right)-\rho\left(x_{1}, x_{3}\right) \rho\left(x_{4}, x_{2}\right)+\rho\left(x_{1}, x_{2}\right) \rho\left(x_{4}, x_{3}\right) \\
& -\beta\left(x_{4}, \mu\left(x_{1}, x_{2}, x_{3}\right)\right)-\rho\left(x_{2}, x_{4}\right) \rho\left(x_{3}, x_{1}\right)+\rho\left(x_{1}, x_{4}\right) \rho\left(x_{3}, x_{2}\right) \\
& \quad-\rho\left(x_{1}, x_{2}\right) \rho\left(x_{3}, x_{4}\right)+\beta\left(x_{3}, \mu\left(x_{1}, x_{2}, x_{4}\right)\right) \\
& =\beta\left(x_{3}, \mu\left(x_{1}, x_{2}, x_{4}\right)\right)-\beta\left(x_{4}, \mu\left(x_{1}, x_{2}, x_{3}\right)\right)-\rho\left(x_{2}, x_{3}\right) \rho\left(x_{1}, x_{4}\right) \\
& \quad-\rho\left(x_{1}, x_{4}\right) \rho\left(x_{2}, x_{3}\right)+\rho\left(x_{1}, x_{3}\right) \rho\left(x_{2}, x_{4}\right) \\
& \quad+\rho\left(x_{2}, x_{4}\right) \rho\left(x_{1}, x_{3}\right)-2 \rho\left(x_{1}, x_{2}\right) \rho\left(x_{3}, x_{4}\right) .
\end{aligned}
$$

According to Equations (6) and (10), 


$$
\begin{aligned}
& {\left[x_{1}, x_{2},\left[x_{3}, x_{4}, h\right]_{A}\right]_{A}} \\
& =\left[\left[x_{1}, x_{2}, x_{3}\right]_{A}, x_{4}, h\right]_{A}+\left[x_{3},\left[x_{1}, x_{2}, x_{4}\right]_{A}, h\right]_{A}+\left[x_{3}, x_{4},\left[x_{1}, x_{2}, h\right]_{A}\right]_{A} .
\end{aligned}
$$

Case 3. For all $x_{i} \in M, i=1,2,3, h_{1}, h_{2} \in H$, it is obtained from Equations (15), (16)

$$
\begin{aligned}
& {\left[x_{1}, h_{1},\left[x_{2}, x_{3}, h_{2}\right]_{A}\right]_{A}} \\
& =\left[\left[x_{1}, h_{1}, x_{2}\right]_{A}, x_{3}, h_{2}\right]_{A}+\left[x_{2},\left[x_{1}, h_{1}, x_{3}\right]_{A}, h_{2}\right]_{A}+\left[x_{2}, x_{3},\left[x_{1}, h_{1}, h_{2}\right]_{A}\right]_{A}, \\
& {\left[h_{1}, h_{2},\left[x_{1}, x_{2}, x_{3}\right]_{A}\right]_{A}} \\
& =\left[\left[h_{1}, h_{2}, x_{1}\right]_{A}, x_{2}, x_{3}\right]_{A}+\left[x_{1},\left[h_{1}, h_{2}, x_{2}\right]_{A}, x_{3}\right]_{A}+\left[x_{1}, x_{2},\left[h_{1}, h_{2}, x_{3}\right]_{A}\right]_{A} .
\end{aligned}
$$

Because $\left[x_{1}, x_{2},\left[x_{3}, h_{1}, h_{2}\right]_{A}\right]_{A}=\rho\left(x_{1}, x_{2}\right) \rho\left(x_{3}, h_{1}\right) h_{2}$,

$$
\begin{aligned}
& {\left[\left[x_{1}, x_{2}, x_{3}\right]_{A}, h_{1}, h_{2}\right]_{A}+\left[x_{3},\left[x_{1}, x_{2}, h_{1}\right]_{A}, h_{2}\right]_{A}+\left[x_{3}, h_{1},\left[x_{1}, x_{2}, h_{2}\right]_{A}\right]_{A}} \\
& =\beta\left(\left[x_{1}, x_{2}, x_{3}\right], h_{1}\right) h_{2}+\beta\left(x_{3}, \rho\left(x_{1}, x_{2}\right) h_{1}\right) h_{2} \\
& \quad+\beta\left(x_{3}, h_{1}\right) \rho\left(x_{1}, x_{2}\right) h_{2}+\left[\mu\left(x_{1}, x_{2}, x_{3}\right), h_{1}, h_{2}\right] .
\end{aligned}
$$

Through the direct calculation of Equations (15) and (16),

$$
\begin{aligned}
& {\left[\mu\left(x_{1}, x_{2}, x_{3}\right), h_{1}, h_{2}\right]} \\
& =\beta\left(x_{1}, h_{1}\right) \rho\left(x_{2}, x_{3}\right) h_{2}+\beta\left(x_{3}, h_{2}\right) \rho\left(x_{1}, x_{2}\right) h_{1}-\beta\left(x_{2}, h_{2}\right) \rho\left(x_{1}, x_{3}\right) h_{1} \\
& +\rho\left(x_{1}, x_{3}\right) \beta\left(x_{2}, h_{2}\right) h_{1}-\rho\left(x_{1}, x_{2}\right) \beta\left(x_{3}, h_{2}\right) h_{1}-\beta\left(\left[x_{1}, x_{2}, x_{3}\right], h_{1}\right) h_{2} \\
& {\left[\mu\left(x_{1}, x_{2}, x_{3}\right), h_{1}, h_{2}\right]} \\
& =\beta\left(x_{2}, h_{2}\right) \rho\left(x_{1}, x_{3}\right) h_{1}+\beta\left(x_{3}, h_{1}\right) \rho\left(x_{2}, x_{1}\right) h_{2}-\beta\left(x_{1}, h_{1}\right) \rho\left(x_{2}, x_{3}\right) h_{2} \\
& +\rho\left(x_{2}, x_{3}\right) \beta\left(x_{1}, h_{1}\right) h_{2}-\rho\left(x_{2}, x_{1}\right) \beta\left(x_{3}, h_{1}\right) h_{2}-\beta\left(\left[x_{1}, x_{2}, x_{3}\right], h_{1}\right) h_{2} \\
& {\left[\mu\left(x_{1}, x_{2}, x_{3}\right), h_{1}, h_{2}\right]=\rho\left(x_{1}, x_{2}\right) \beta\left(x_{3}, h_{1}\right) h_{2}-\beta\left(x_{3}, h_{1}\right) \rho\left(x_{1}, x_{2}\right) h_{2}} \\
& +\beta\left(x_{3}, h_{2}\right) \rho\left(x_{1}, x_{2}\right) h_{1}-\beta\left(\left[x_{1}, x_{2}, x_{3}\right], h_{1}\right) h_{2} .
\end{aligned}
$$

As a result,

$$
\begin{aligned}
& {\left[x_{1}, x_{2},\left[x_{3}, h_{1}, h_{2}\right]_{A}\right]_{A}} \\
& =\left[\left[x_{1}, x_{2}, x_{3}\right]_{A}, h_{1}, h_{2}\right]_{A}+\left[x_{3},\left[x_{1}, x_{2}, h_{1}\right]_{A}, h_{2}\right]_{A}+\left[x_{3}, h_{1},\left[x_{1}, x_{2}, h_{2}\right]_{A}\right]_{A} .
\end{aligned}
$$

Case 4. For all $x_{1}, x_{2} \in M, h_{i} \in H, i=1,2,3$, due to the $\beta\left(x_{1}, x_{2}\right) \in \operatorname{Der}(H)$, it can be concluded from Equation (11) that,

$$
\begin{aligned}
& {\left[x_{1}, x_{2},\left[h_{1}, h_{2}, h_{3}\right]_{A}\right]_{A}} \\
& =\left[\left[x_{1}, x_{2}, h_{1}\right]_{A}, h_{2}, h_{3}\right]_{A}+\left[h_{1},\left[x_{1}, x_{2}, h_{2}\right]_{A}, h_{3}\right]_{A}+\left[h_{1}, h_{2},\left[x_{1}, x_{2}, h_{3}\right]_{A}\right]_{A} \\
& {\left[x_{1}, h_{1},\left[x_{2}, h_{2}, h_{3}\right]_{A}\right]_{A}} \\
& =\left[\left[x_{1}, h_{1}, x_{2}\right]_{A}, h_{2}, h_{3}\right]_{A}+\left[x_{2},\left[x_{1}, h_{1}, h_{2}\right]_{A}, h_{3}\right]_{A}+\left[x_{2}, h_{2},\left[x_{1}, h_{1}, h_{3}\right]_{A}\right]_{A}
\end{aligned}
$$

Because of $\left[h_{1}, h_{2},\left[h_{3}, x_{1}, x_{2}\right]_{A}\right]_{A}=\left[h_{1}, h_{2}, \rho\left(x_{1}, x_{2}\right) h_{3}\right]$,

Then

$$
\begin{aligned}
& {\left[\left[h_{1}, h_{2}, h_{3}\right]_{A}, x_{1}, x_{2}\right]_{A}+\left[h_{3},\left[h_{1}, h_{2}, x_{1}\right]_{A}, x_{2}\right]_{A}+\left[h_{3}, x_{1},\left[h_{1}, h_{2}, x_{2}\right]_{A}\right]_{A}} \\
& =\rho\left(x_{1}, x_{2}\right)\left[h_{1}, h_{2}, h_{3}\right]+\beta\left(x_{2}, h_{3}\right) \beta\left(x_{1}, h_{1}\right) h_{2}-\beta\left(x_{1}, h_{3}\right) \beta\left(x_{2}, h_{1}\right) h_{2}
\end{aligned}
$$


According to lemma 4.2,

$$
\begin{aligned}
& \rho\left(x_{1}, x_{2}\right)\left[h_{1}, h_{2}, h_{3}\right] \\
& =\beta\left(x_{2}, h_{1}\right) \beta\left(x_{1}, h_{3}\right) h_{2}+\beta\left(x_{2}, h_{2}\right) \beta\left(x_{1}, h_{1}\right) h_{3}+\beta\left(x_{2}, h_{3}\right) \beta\left(x_{1}, h_{2}\right) h_{1} \\
& \beta\left(x_{2}, h_{2}\right) \beta\left(x_{1}, h_{1}\right) h_{3}-\beta\left(x_{2}, h_{3}\right) \beta\left(x_{1}, h_{1}\right) h_{2} \\
& =\rho\left(x_{1}, x_{2}\right)\left[h_{1}, h_{2}, h_{3}\right]+\beta\left(x_{2}, h_{1}\right) \beta\left(x_{1}, h_{2}\right) h_{3}
\end{aligned}
$$

Using Equation (11) again, we can get

$$
\begin{aligned}
& {\left[\rho\left(x_{1}, x_{2}\right) h_{1}, h_{2}, h_{3}\right]} \\
& =\rho\left(x_{1}, x_{2}\right)\left[h_{1}, h_{2}, h_{3}\right]+\beta\left(x_{2}, h_{1}\right) \beta\left(x_{1}, h_{2}\right) h_{3}-\beta\left(x_{1}, h_{1}\right) \beta\left(x_{2}, h_{2}\right) h_{3}
\end{aligned}
$$

namely

$$
\begin{aligned}
& {\left[h_{1}, h_{2},\left[h_{3}, x_{1}, x_{2}\right]_{A}\right]_{A}} \\
& =\left[\left[h_{1}, h_{2}, h_{3}\right]_{A}, x_{1}, x_{2}\right]_{A}+\left[h_{3},\left[h_{1}, h_{2}, x_{1}\right]_{A}, x_{2}\right]_{A}+\left[h_{3}, x_{1},\left[h_{1}, h_{2}, x_{2}\right]_{A}\right]_{A} .
\end{aligned}
$$

Case 5. For all $x \in M, h_{i} \in H, i=1,2,3,4$, because $\beta\left(x, h_{1}\right) \in \operatorname{Der}(H)$, through Equation (13),

$$
\begin{aligned}
& {\left[x, h_{1},\left[h_{2}, h_{3}, h_{4}\right]_{A}\right]_{A}} \\
& =\left[\left[x, h_{1}, h_{2}\right]_{A}, h_{3}, h_{4}\right]_{A}+\left[h_{2},\left[x, h_{1}, h_{3}\right]_{A}, h_{4}\right]_{A}+\left[h_{2}, h_{3},\left[x, h_{1}, h_{4}\right]_{A}\right]_{A} \\
& {\left[h_{1}, h_{2},\left[h_{3}, h_{4}, x\right]_{A}\right]_{A}} \\
& =\left[\left[h_{2}, h_{3}, h_{4}\right]_{A}, h_{4}, x\right]_{A}+\left[h_{3},\left[h_{1}, h_{3}, h_{4}\right]_{A}, x\right]_{A}+\left[h_{3}, h_{4},\left[h_{1}, h_{2}, x\right]_{A}\right]_{A}
\end{aligned}
$$

To sum up, (8) satisfies Equation (1). The conclusion is proved.

Theorem 4.2 Let $A=M \oplus H$ be the $(\mu, \rho, \beta)$-extension of 3-Lie algebra $H$ through $M$. So $(H, \rho)$ is $M$-module if and only if $\beta(M, \mu(M, M, M))=0$.

Proof: If $\beta(M, \mu(M, M, M))=0$, obviously $(H, \rho)$ is an $M$-module.

On the other hand, to any $x_{j} \in M, j=1,2,3,4$, by theorem 4.1 and Equation (9), (10),

$$
\begin{gathered}
\rho\left(\left[x_{1}, x_{2}, x_{4}\right], x_{3}\right)-\rho\left(\left[x_{1}, x_{2}, x_{3}\right], x_{4}\right) \\
=-\beta\left(x_{4}, \mu\left(x_{1}, x_{2}, x_{3}\right)\right)+\beta\left(x_{3}, \mu\left(x_{1}, x_{2}, x_{4}\right)\right) \\
-\rho\left(x_{1}, x_{2}\right) \rho\left(x_{3}, x_{4}\right)-\rho\left(x_{3}, x_{4}\right) \rho\left(x_{1}, x_{2}\right) \\
\rho\left(x_{2},\left[x_{1}, x_{3}, x_{4}\right]\right)=\rho\left(x_{3}, x_{4}\right) \rho\left(x_{2}, x_{1}\right)-\rho\left(x_{1}, x_{4}\right) \rho\left(x_{2}, x_{3}\right) \\
+\rho\left(x_{1}, x_{3}\right) \rho\left(x_{2}, x_{4}\right)-\beta\left(x_{2}, \mu\left(x_{1}, x_{3}, x_{4}\right)\right)
\end{gathered}
$$

As a result,

$$
\begin{aligned}
& -\rho\left(\left[x_{1}, x_{2}, x_{3}\right], x_{4}\right)+\rho\left(\left[x_{1}, x_{2}, x_{4}\right], x_{3}\right)+\rho\left(x_{2},\left[x_{1}, x_{3}, x_{4}\right]\right)-\rho\left(x_{1},\left[x_{2}, x_{3}, x_{4}\right]\right) \\
& =-\beta\left(x_{4}, \mu\left(x_{1}, x_{2}, x_{3}\right)\right)+\beta\left(x_{3}, \mu\left(x_{1}, x_{2}, x_{4}\right)\right)-\rho\left(x_{1}, x_{2}\right) \rho\left(x_{3}, x_{4}\right) \\
& +\rho\left(x_{3}, x_{4}\right) \rho\left(x_{1}, x_{2}\right)+\rho\left(x_{3}, x_{4}\right) \rho\left(x_{2}, x_{1}\right)-\rho\left(x_{1}, x_{4}\right) \rho\left(x_{2}, x_{3}\right) \\
& +\rho\left(x_{1}, x_{3}\right) \rho\left(x_{2}, x_{4}\right)-\beta\left(x_{2}, \mu\left(x_{1}, x_{3}, x_{4}\right)\right)-\rho\left(x_{3}, x_{4}\right) \rho\left(x_{1}, x_{2}\right) \\
& +\rho\left(x_{2}, x_{4}\right) \rho\left(x_{1}, x_{3}\right)-\rho\left(x_{2}, x_{3}\right) \rho\left(x_{1}, x_{4}\right)+\beta\left(x_{1}, \mu\left(x_{2}, x_{3}, x_{4}\right)\right)
\end{aligned}
$$




$$
\begin{aligned}
= & \beta\left(x_{3}, \mu\left(x_{1}, x_{2}, x_{4}\right)\right)-\beta\left(x_{4}, \mu\left(x_{1}, x_{2}, x_{3}\right)\right)+\beta\left(x_{1}, \mu\left(x_{2}, x_{3}, x_{4}\right)\right) \\
& -\beta\left(x_{2}, \mu\left(x_{1}, x_{3}, x_{4}\right)\right)-\rho\left(x_{1}, x_{2}\right) \rho\left(x_{3}, x_{4}\right)-\rho\left(x_{3}, x_{4}\right) \rho\left(x_{1}, x_{2}\right) \\
& +\rho\left(x_{1}, x_{3}\right) \rho\left(x_{2}, x_{4}\right)+\rho\left(x_{2}, x_{4}\right) \rho\left(x_{1}, x_{3}\right)-\rho\left(x_{1}, x_{4}\right) \rho\left(x_{2}, x_{3}\right) \\
& -\rho\left(x_{2}, x_{3}\right) \rho\left(x_{1}, x_{4}\right) \\
= & \beta\left(x_{3}, \mu\left(x_{1}, x_{2}, x_{4}\right)\right)-\beta\left(x_{4}, \mu\left(x_{1}, x_{2}, x_{3}\right)\right) \\
& +\beta\left(x_{1}, \mu\left(x_{2}, x_{3}, x_{4}\right)\right)-\beta\left(x_{2}, \mu\left(x_{1}, x_{3}, x_{4}\right)\right) \\
= & 0
\end{aligned}
$$

According to Equation (9), $\beta\left(x_{4}, \mu\left(x_{1}, x_{2}, x_{3}\right)\right)=0$. And the theorem is proved.

Theorem 4.3 Let $A=M \oplus H$ be the $(H, \rho)$-extension of 3-Lie algebra $H$ through $M$ and $(H, \rho)$ be an $M$-module. So $A$ is a 3-Lie algebra if and only if $\mu(M, M, M) \subseteq Z(H), \rho(M, M) \subseteq Z(\operatorname{Der} H)$ and Equation (17) is true.

Proof: If $A$ is a 3-Lie algebra, Equation (17) holds by theorem 4.1. Since $(H, \rho)$ is $M$-module, then $\beta=0$. And $\mu(M, M, M) \subseteq Z(H)$, $\rho(M, M) \subseteq Z(\operatorname{DerH})$ can be obtained by Equations (9) and (13). Conversely, from theorems 4.1 and 4.2, $A$ is a 3 -Lie algebra.

The above conclusions about 3-Lie algebras will be helpful for further study of their derivation algebras.

\section{Funding}

Science and Technology Research Project of Higher Education Department of Hebei Province (Z2015009).

\section{Conflicts of Interest}

The authors declare no conflicts of interest regarding the publication of this paper.

\section{References}

[1] Filippov, V.T. (1985) n-Lie Algebras. Siberian Mathematical Journal, 26, 126-140. https://doi.org/10.1007/BF00969110

[2] Lu, F.Y. (2007) Lie Triple Derivations on Nest Algebras. Mathematische Nachrichten, 280, 882-887. https://doi.org/10.1002/mana.200410520

[3] Papageorgakis, C. and Samann, C. (2011) The 3-Lie Algebra (2, 0) Tensor Multiplet and Equations of Motion on Loop Space. Journal of High Energy Physics, 2011, Article No. 99. https://doi.org/10.1007/JHEP05(2011)099

[4] Bremner, M. and Elgendy, H. (1987) Universal Associative Envelopes of $(n+$ 1)-Dimensional $n$-Lie Algebras.

[5] Bai, R.P., Bai, C.M. and Wang, J.X. (2010) Realizations of 3-Lie Algebras. Journal of Mathematical Physics, 51, Article ID: 063505. https://doi.org/10.1063/1.3436555

[6] Bai, R. and Wu, Y. (2015) Constructions of 3-Lie Algebras. Linear and Multilinear Algebra, 63, 2171-2186. https://doi.org/10.1080/03081087.2014.986121

[7] Bai, R., Wu, W. and Li, Z. (2012) Some Results on Metric n-Lie Algebras. Acta Mathematics Sinica, English Series, 28, 1209-1220.

https://doi.org/10.1007/s10114-011-0231-4 
[8] Bai, R., Chen, S. and Cheng, R. (2016) Symplectic Structures on 3-Lie Algebras.

[9] Bai, R., Wu, W. and Li, Y. (2012) Module Extensions of 3-Lie Algebras. Linear and Multilinear Algebra, 60, 433-447. https://doi.org/10.1080/03081087.2011.603728 